\title{
Heavy Smoker
}

National Cancer Institute

\section{Source}

National Cancer Institute. Heavy Smoker. NCI Thesaurus. Code C154510.

A smoker who smokes more than 10 cig arettes per day, or an equivalent quantity of cigar or pipe smoke. 